\title{
A Co-Design Methodology for Energy-Efficient Multi-Mode Embedded Systems with Consideration of Mode Execution Probabilities
}

\author{
Marcus T. Schmitz and Bashir M. Al-Hashimi \\ Dept. of Electronics and Computer Science \\ University of Southampton \\ Southampton, SO17 1BJ, United Kingdom \\ \{m.schmitz,bmah\}@ecs.soton.ac.uk
}

\begin{abstract}
Multi-mode systems are characterised by a set of interacting operational modes to support different functionalities and standards. In this paper, we present a co-design methodology for multi-mode embedded systems that produces energy-efficient implementations. Based on the key observation that operational modes are executed with different probabilities, i.e., the system spends uneven amounts of time in the different modes, we develop a novel co-design technique that exploits this property to significantly reduce energy dissipation. We conduct several experiments, including a smart phone real-life example, that demonstrate the effectiveness of our approach. Reductions in power consumption of up to $64 \%$ are reported.
\end{abstract}

\section{Introduction}

The need for embedded systems continues to increase and power consumption has become one of the most important design objectives. Over the last few year numerous methodologies for the design of low power consuming embedded systems have been proposed, among which we find approaches that leverage power management techniques, such as dynamic power management (DPM) and dynamic voltage scaling (DVS).

A key characteristic of many current and emerging embedded systems is their need to work across a set of different interacting operational modes. Such systems are called multi-mode embedded systems. Three previous approaches have addressed the problem of designing mixed hardware/software implementations of multi-mode systems $[7,9,13]$. The main principle behind these approaches is to consider the possibility of hardware sharing, i.e., computational tasks of the same type, which can be found in different modes, utilise the same hardware component. Thereby, multiple implementations of the same task type are avoided, which, in turn, reduces the necessary hardware cost.

As opposed to these approaches, the presented work addresses the design of low energy consuming multi-mode systems, hence, it differs in several aspects from the previous works. The paper makes the following contributions: a) We analyse the effects that the consideration of mode execution probabilities has on energy-efficiency. b) A co-design methodology for the design of energy-efficient multi-mode systems is presented. We propose a co-synthesis for multi-mode systems which maps and schedules a system specification that captures both mode interaction and mode functionality. c) We investigate dynamic voltage scaling in the context of multi-mode systems and consider that not only programmable processors might be DVS enabled, but additionally the hardware components.

\author{
Petru Eles \\ Dept. of Computer and Information Science \\ Linköping University \\ S-58183 Linköping, Sweden \\ petel@ida.liu.se
}

\section{Preliminaries}

\subsection{Functional Specification of Multi-Mode Systems}

The abstract specification model we consider here is based on a combination of finite state machines and task graphs, used to capture the interaction between different operational modes as well as the functionality of each individual mode. We refer to this model as operational mode state machine (OMSM). A similar model was used in [4]. However, we extend this model towards system-level design and include transition time limits as well as mode execution probabilities. The following section explains this model, using the smart phone example shown in Fig. 1a). This smart phone combines three different functionalities within one device: a GSM phone, a digital camera, and an MP3-player.

\subsubsection{Top-level Finite State Machine}

We consider that an application is given as a directed cyclic graph $\Upsilon(\Omega, \Theta)$, which represents a finite state machine. Within this toplevel model, each node $O \in \Omega$ refers to an operational mode and each edge $T \in \Theta$ specifies a transition between two modes. If the system undergoes a change from mode $O_{x}$ to mode $O_{y}$, with $x \neq y$, the transition time $t_{T}^{\max }$ associated with the transition edge $T=\left(O_{x}, O_{y}\right)$ has to be met. At any given time there is only one active mode, i.e., the modes are mutually exclusive. Fig. 1a) exemplifies the operational mode state machine for a smart phone example with eight different modes. An activation scenario could look like this: When switched on, the phone initialises into Network Search mode. Upon finding a network, the phone changes into Radio Link Control (RLC) mode. In this mode it maintains the connection to the network by handling cell handovers, radio link failure responses, and adaptive RF power control. An incoming phone call necessitates to switch into GSM codec + RLC mode. This mode is responsible for speech encoding and decoding while maintaining network connectivity. Similarly, the remaining modes have different functions and are activated upon mode change events. Such events originate upon user requests (e.g. MP3-player activation) or are initiated by the system itself (e.g. loss of network-switch back into network search mode).

Based on the key observation that many multi-mode systems spend their operational time not evenly in each of the modes, we assume that for each operational mode $O$ its execution probability $\Psi_{O}$ is given, i.e., we know what percentage of the operational time the device spends in each mode. For instance, in accordance to Fig. 1 a), the smart phone stays $74 \%$ of this operational time in Radio Link Control (RLC) mode, $9 \%$ in GSM codec + RLC mode, and $1 \%$ in Network Search mode. The left over 16\% of the operation time are associated with the remaining modes. Of course, in reality the mode probabilities vary from user to user. Nevertheless, it is possible to derive an "average" usage profile based 


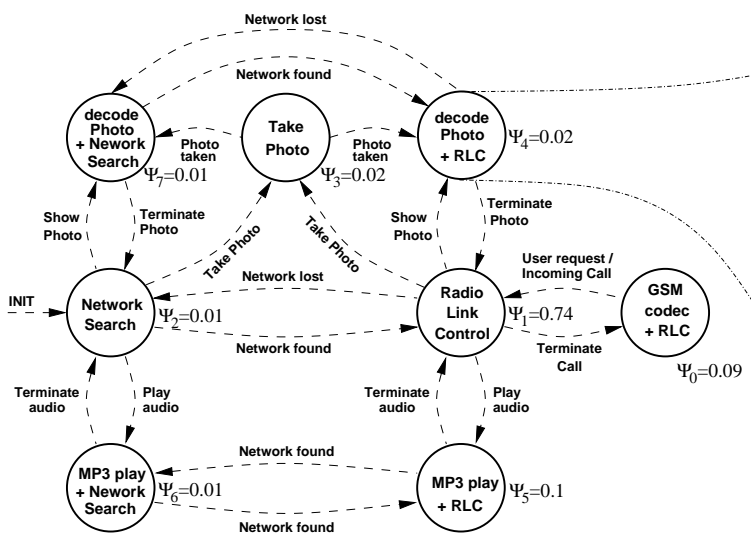

a) Operational Mode State Machine

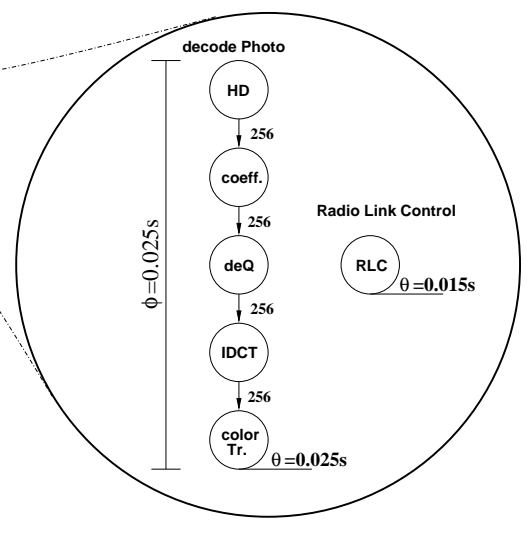

b) Task Graph of a Single Mode

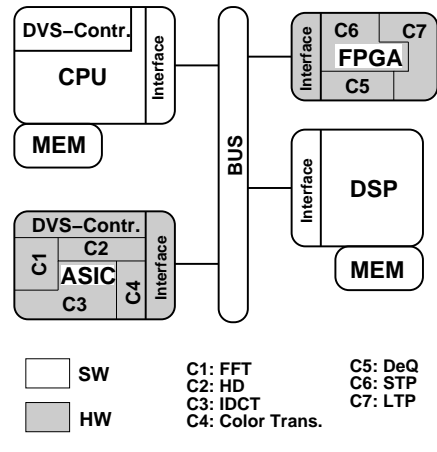

c) Possible Target Architecture

Figure 1. Architectural and Specification Model

on statistical information collected from several different users. Taking this information into account will prove to be important when designing systems with a prolonged battery life-time.

\subsubsection{Functional Specification of Individual Modes}

The functional specification of each mode $O \in \Omega$ in the top-level finite state machine is expressed by a task graph $G_{S}^{O}(\mathcal{T}, \mathcal{C})$; see Fig. 1b). Here, each node $\tau \in \mathcal{T}$ represents a task, an atomic unit of functionality that needs to be executed without preemption. We consider a coarse level of granularity where tasks refer to functions such as Huffman decoders, de-quantizers, FFTs, IDCTs, etc. Therefore, each task is further associated with a task type $\eta \in \Gamma^{O}$. A distinctive feature of multi-mode systems is that task type sets $\Gamma^{O}$ of different modes $O \in \Omega$ can intersect, i.e., tasks of identical type can share the same hardware resource. Of course, resource sharing is also possible for multiple tasks of identical type which are found in a single mode, however, due to task communalities among different modes the chances to share resources are increased. Edges $\gamma \in \mathcal{C}$ in the task graph refer to precedence constraints and data dependencies between the computational tasks, i.e., if two tasks, $\tau_{i}$ and $\tau_{j}$, are connected by an edge, then task $\tau_{i}$ must have finished and transfered its data to task $\tau_{j}$, before $\tau_{j}$ can be executed. A feasible implementation needs to obey all timing constraints and precedence relations.

\subsection{Architectural Model and System Implementation}

Our system-level synthesis approach targets distributed architectures that possibly consist of several heterogeneous processing elements (PEs), such as general purpose processors (GPPs), ASIPs, ASICs, and FPGAs. These components are connected through an infrastructure of communication links (CLs). A directed graph $G_{A}(\mathcal{P}, \mathcal{L})$ captures such an architecture, where nodes $\pi \in \mathcal{P}$ and edges $\lambda \in \mathcal{L}$ denote PEs and CLs, respectively; Fig. 1c) shows an example architecture.

Since each task might have multiple implementation alternatives, it can be potentially mapped onto several different PEs that are capable to perform this type of task. However, if a task is mapped to a hardware component, i.e., ASIC or FPGA, a core for this task type needs to be allocated, involving the usage of area. Tasks assigned to GPPs or ASIPs (software tasks) need to be sequentialised whilst the tasks mapped onto FPGAs and ASICs (hardware tasks) can be performed in parallel if the necessary resources (cores) are not already engaged. However, contention between two or more tasks assigned to the same hardware core necessitates a sequential execution order, similar to software tasks. Cores implemented on FPGAs can be dynamically reconfigured during a mode change, involving a time overhead which needs to respect the imposed maximal mode transition times.

Further, PEs might feature dynamic voltage scaling to enable a tradeoff between power consumption and performance which can be exploited during run-time. For such PEs a voltage schedule needs to be derived, additionally to a timing schedule [10].

To implement a multi-mode application captured as OMSM, the tasks and communications of all operational modes need to be mapped onto the architecture, and a valid schedule for these activities $\varepsilon \in(\mathcal{A}=\mathcal{T} \cup \mathcal{C})$ needs to be constructed. Further, for tasks mapped to DVS enabled components an energy reducing voltage schedule has to be determined. Hence, an implementation candidate can be expressed through four functions which need to be derived for each operational mode $O \in \Omega: M_{\tau}^{O}: \mathcal{T} \rightarrow \pi, M_{\gamma}^{O}: \mathcal{C} \rightarrow \lambda$, $S_{\varepsilon}^{O}: \mathcal{A} \rightarrow \mathbb{R}_{0}^{+}$, and $V_{\tau}^{O}: \mathcal{T}_{D V S} \rightarrow \mathcal{V}_{\pi}$, where $M_{\tau}^{O}$ and $M_{\gamma}^{O}$ denote task and communication mapping, respectively. Activity start times are specified by the scheduling function $S_{\varepsilon}^{O}$, while $V_{\tau}^{O}$ defines the voltage schedule for all tasks $\tau \in \mathcal{T}_{D V S}$ mapped to DVSPEs, where $\mathcal{V}_{\pi}$ is the set of the possible discrete supply voltages of PE $\pi$. Clearly, the mappings as well as the corresponding schedules are defined for every mode separately, i.e., during the change from mode $O_{x}$ to mode $O_{y}$, the execution of activities found in mode $O_{x}$ are finished, and the activities of mode $O_{y}$ are activated.

\subsection{Motivational Example}

The influence of mapping in the context of multi-mode systems with different mode execution probabilities is demonstrated in the following example. For simplicity we neglect timing and communication issues here. Consider the application shown in Fig. 2 a) which consists of two operational modes, $\mathrm{O}_{1}$ and $\mathrm{O}_{2}$, each specified by a task graph with three tasks. The system spends $10 \%$ of its time in mode $O_{1}$ and the remaining $90 \%$ in mode $O_{2}$, i.e., the execution probabilities are given by $\Psi_{1}=0.1$ and $\Psi_{2}=0.9$. The specification needs to be mapped onto an architecture built of a general purpose processor (PE0) and an ASIC (PE1), linked by a bus (CL0). Depending on the task mapping to either of the components, the execution properties of each task are given in the following table. It can be observed that all tasks are of different

\begin{tabular}{c||c|c||c|c|c}
\hline \multirow{2}{*}{$\begin{array}{c}\text { task } \\
\text { type }\end{array}$} & \multicolumn{2}{c||}{ PE0 (SW) } & \multicolumn{3}{c}{ PE1 (HW) } \\
\cline { 2 - 6 } time $(m s)$ & $\begin{array}{c}\text { dyn. energy } \\
(\mathrm{mWs})\end{array}$ & $\begin{array}{c}\text { exec. } \\
\text { time }(\mathrm{ms})\end{array}$ & $\begin{array}{c}\text { dyn. energy } \\
(\mathrm{mWs})\end{array}$ & $\begin{array}{c}\text { area } \\
(\text { cells })\end{array}$ \\
\hline \hline $\mathrm{A}$ & 20 & 10 & 2 & 0.010 & 240 \\
\hline $\mathrm{B}$ & 28 & 14 & 2.2 & 0.012 & 300 \\
\hline $\mathrm{C}$ & 32 & 16 & 1.6 & 0.023 & 275 \\
\hline $\mathrm{D}$ & 26 & 13 & 3.1 & 0.047 & 245 \\
\hline $\mathrm{E}$ & 30 & 15 & 1.8 & 0.015 & 210 \\
\hline $\mathrm{F}$ & 24 & 14 & 2.2 & 0.032 & 280 \\
\hline
\end{tabular}

type, therefore, if a task is mapped to $\mathrm{HW}$, a suitable core needs to 


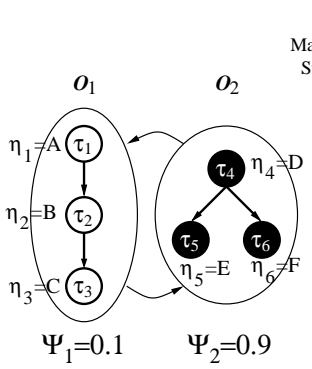

a) Application specified by two interacting modes
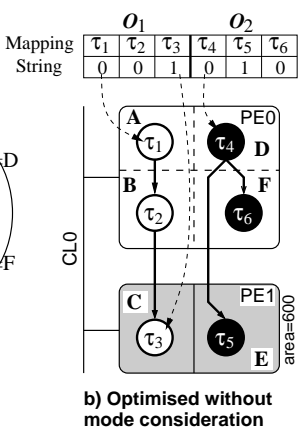
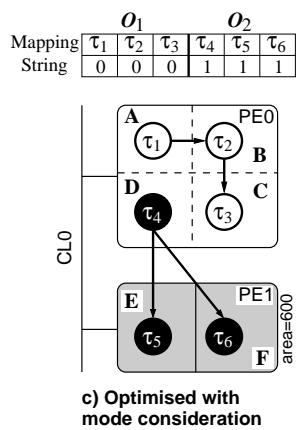

Figure 2. Example 1: Mode Execution Probabilities

be allocated explicitly for that task. Hence, in this particular example, we do not consider hardware sharing. Each allocated core uses area on the hardware component which offers 600 cells, i.e., at most 2 cores can be allocated at the same time without violating the area constraint (see above table, column 6). Note, although the two modes execute mutually exclusive, the task types implemented in hardware (cores) cannot be changed during run-time, since their implementation is static (non-reconfigurable ASIC).

Consider the mapping shown in Fig. 2b). This represents the optimal solution in terms of energy dissipation when neglecting the execution probabilities, since the highest energy dissipating task $\left(\tau_{3}\right.$ and $\left.\tau_{5}\right)$ are executed using a more energyefficient hardware implementation. Nevertheless, taking the real behaviour into account, mode $O_{1}$ is active for $10 \%$ of the operational time, i.e., its energy dissipation can then be calculated as $0.1 \cdot(10 \mathrm{mWs}+14 \mathrm{mWs}+23 \mu W s)=2.4023 \mathrm{mWs}$. Similarly, mode $O_{2}$ is active $90 \%$ of the time, hence, its energy is given by $0.9 \cdot(13 m W s+15 \mu W s+14 m W s)=24.3135 m W s$. Based on both modes, the total energy dissipation results in $26.7158 \mathrm{mWs}$.

Fig. 2c) shows an alternative mapping which represents the optimal assignment of tasks when mode execution probabilities are considered. In this configuration tasks $\tau_{5}$ and $\tau_{6}$ use a hardware implementation. According to this solution, the energy dissipation of mode 1 and mode 2 are given by $0.1 \cdot(10 m W s+14 m W s+16 m W s)=4 m W s$ and $0.9 \cdot(13 m W s+$ $15 \mu W s+32 \mu W s)=11.7423 \mathrm{mWs}$, respectively. The total energy for this mapping is $15.7423 \mathrm{mWs}$, hence, it is $41 \%$ lower compared to the first mapping shown in Fig. 2b), which is not optimised for an uneven task execution probability. Furthermore, the second task mapping, shown in Fig. 2c), allows to switch-off PE1 and CL0 during mode $O_{1}$, since all tasks of this mode are assigned to PE0. This results in a significant reduction of the static power, further increasing the energy savings.

An important characteristic of multi-mode systems is that tasks of the same type might be found in different operational modes, i.e., resources can be shared among the different modes. To increase the possibility of component shut-down, it might be necessary to implement the same task type multiple times, however, on different components. The following example, shown in Fig. 3, clarifies this aspect. Here tasks $\tau_{1}$ and $\tau_{4}$ are of type A, allowing resource sharing. In the first mapping, given in Fig. 3 b), both tasks utilise the same HW core. However, additionally implementing task $\tau_{4}$ as software, as shown in Fig. 3c), allows to shut-down PE1 and CL0 during the execution of mode $\mathrm{O}_{2}$. Hence, multiple implementations of task types can help to reduce power dissipation.

These two examples show that it is essential to guide the synthesis process by an energy model that takes into account the execution probability and allows multiple task implementations.

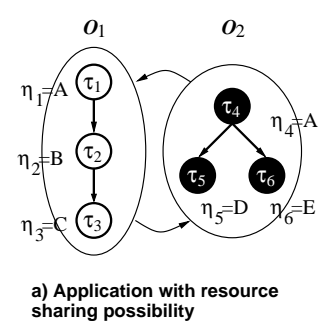

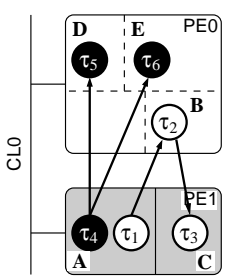

b) Resource sharing, but no shut-down possib
butce sharing,

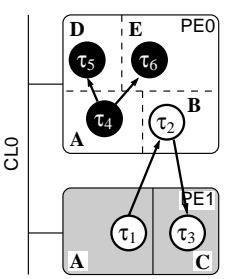

c) No resource sharing

but component shut-down
Figure 3. Example 2: Multiple Task Implementations

\section{Problem Formulation}

Our goal is an energy-efficient and feasible implementation of application $\Upsilon$, modelled as an OMSM. This involves the derivation of the mapping and schedule functions $\left(M_{\tau}^{O}, M_{\gamma}^{O}, S_{\varepsilon}^{O}\right.$, and $\left.V_{\tau}^{O}\right)$, see Section 2.2, under the consideration of static and dynamic power dissipations as well as mode execution probabilities. The average power consumption $\bar{p}$ of an implementation alternative is given by:

$$
\bar{p}=\sum_{O \in \Omega}\left(\bar{p}_{O}^{d y n}+\bar{p}_{O}^{s t a t}\right) \cdot \Psi_{O}
$$

where $\bar{p}_{O}^{d y n}, \bar{p}_{O}^{\text {stat }}$, and $\Psi_{O}$ refer to the dynamic power consumption, the static power consumption, and execution probability of mode $O$, respectively. The power consumptions are given as,

$$
\bar{p}_{O}^{d y n}=\left(\sum_{\varepsilon \in \mathcal{A}_{O}} E(\varepsilon)\right) \cdot \frac{1}{h p_{O}} \text { and } \bar{p}_{O}^{\text {stat }}=\sum_{\xi \in \mathcal{K}_{O}} \bar{P}^{\text {stat }}(\xi)
$$

where $\mathcal{A}_{O}$ and $h p_{O}$ are all activities and the hyper-period of mode $O$, respectively. $\bar{P}^{\text {stat }}(\xi)$ refers to the static power consumption of a component $\xi$, found in the set of all active components $\mathcal{K}_{0} \subseteq \mathcal{P} \cup \mathcal{L}$ of mode $O$. With respect to the type of activities, the dynamic energy consumption $E(\varepsilon)$ is calculated as,

$$
E(\varepsilon)= \begin{cases}P_{\max }(\varepsilon) \cdot t_{\min }(\varepsilon) \cdot V_{d d}^{2}(\varepsilon) & \text { if } \varepsilon \in \mathcal{T}_{\text {DVS }} \\ P_{\max }(\varepsilon) \cdot t_{\min }(\varepsilon) & \text { if } \varepsilon \in \mathcal{T} \backslash \mathcal{T}_{\text {DVS }} \\ P_{C}(\varepsilon) \cdot t_{C}(\varepsilon) & \text { if } \varepsilon \in \mathcal{C}\end{cases}
$$

where $P_{\max }$ is the dynamic power dissipation and $t_{\min }$ the execution time of tasks when executed at nominal supply voltage $V_{\max }$. Tasks $\tau \in \mathcal{T}_{\text {DVS }}$ mapped to DVS-PEs can execute at a scaled supply voltage $V_{d d}$, resulting in a reduced power consumption. Further, communications consume power $P_{C}$ over a time $t_{C}$.

The synthesis goal is to find a task mapping $M_{\tau}^{O}$, a communication mapping $M_{\gamma}^{O}$, a time schedule $S_{\varepsilon}^{O}$, and a voltage schedule $V_{\tau}^{O}$ for each operational mode $O$, such that the total average power $\bar{p}$, given in Equation (1), is minimised. A feasible implementation candidate needs to fulfil the following requirements: a) The mapping of tasks $M_{\tau}^{O}$ does not violate area constraints, i.e., $\left(\sum_{\eta \in \Gamma_{\pi}} a_{\eta}\right) \leq a_{\pi}^{\max }, \quad \forall \pi \in \mathcal{P}$; where $\Gamma_{\pi}$ is the set of all task types implemented on PE $\pi$, and $a_{\eta}$ and $a_{\pi}^{\max }$ refer to the area used by task type $\eta$ and the available area on PE $\pi$, respectively. b) The timing schedule $S_{\varepsilon}^{O}$ and the voltage schedule $V_{\tau}^{O}$, based on task and communication mapping, do not exceed any task deadlines $\theta_{\tau}$ or task graph repetition periods $\phi$, therefore, $t_{S}(\tau)+t_{\text {exe }}(\tau) \leq \min \left(\theta_{\tau}, \phi\right), \quad \forall \tau \in \mathcal{T}$; where $t_{S}(\tau)$ and $t_{\text {exe }}$ refer to task start time and task execution time (potentially based on voltage scaling), respectively. c) The system reconfiguration time $t_{T}$ between mode changes does not exceed the imposed maximal mode transition times $t_{T}^{\max }$. Hence, $t_{T} \leq t_{T}^{\max }, \forall T \in \Theta$, needs to be respected for all mode transitions.

\section{Synthesis of Low Power Multi-Mode Systems}

Our co-synthesis approach for energy-efficient multi-mode systems is based on two nested optimisation loops. The outer loop 


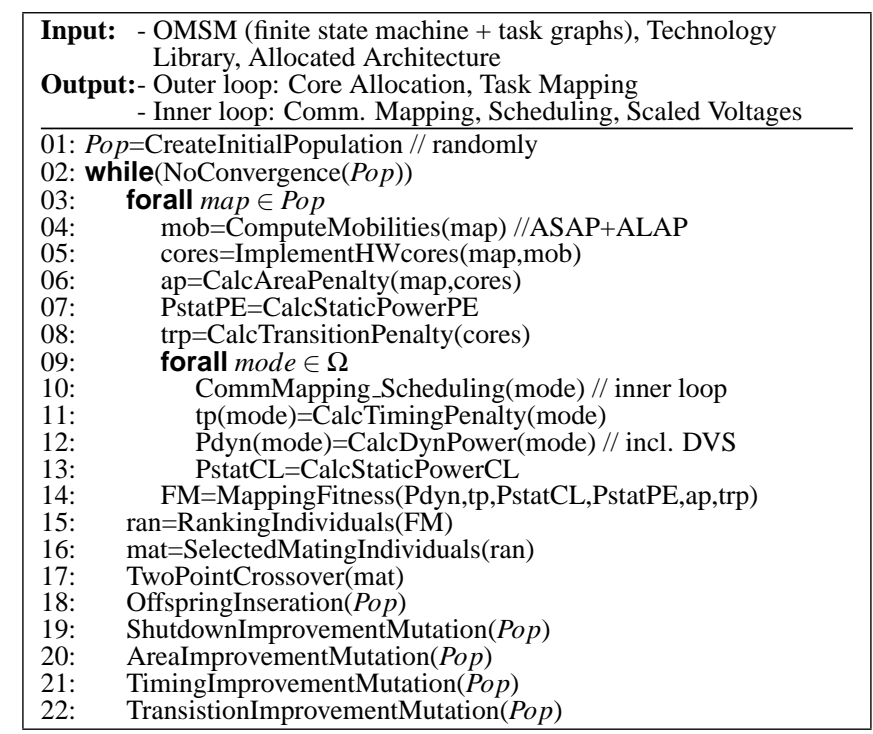

Figure 4. Pseudo Code: Multi-Mode Task Mapping GA

optimises task mapping and core allocation while the inner loop is responsible for the optimisation of communication mapping and scheduling. Due to the space limitations we focus here on the mapping and core allocation, which are explained next, and refer the interested reader to [12] were communication mapping and scheduling are outlined.

\subsection{Task Mapping and Core Allocation Approach}

The presented task mapping approach, which determines $M_{\tau}$ for all modes of application $\Upsilon$, is based on a genetic algorithm (GA). More exactly, we have extended a GA mapping technique for single mode systems [11] to suit the particular problems of multimode systems. These problems include resource sharing, component shut-down, and mode transition issues.

In general, GAs optimise a population of individuals over several generations by imitating and applying the principles of natural selection. I.e., the GA iteratively evolves new populations by mating (crossover) the fittest individuals (highest quality) of the current population pool until a certain convergency criterion is met. In addition to mating, mutation, that is, the random change of genes in the genome (string), provides the opportunity to push the optimisation into unexplored search space regions. An excellent introduction to GA can be found in [3]. In our case, each mapping candidate is encoded into a multi-mode mapping string as shown in Fig. 2b) and Fig. 2c). A detailed description of the proposed mapping GA is outlined in Fig. 4.

Starting from an initial random population (line 1), the optimisation runs until the convergency criterion is met (line 2). The criterion we use is based on the diversity in the current population and the number of elapsed iterations without any improved individual. To judge the quality of mapping candidates, i.e., the fitness which guides the genetic algorithm, it is necessary to estimate important design objectives, including static and dynamic power dissipation, area usage, and timing behaviour (lines 0313). The following explains each of the estimations. The hardware area depends on the allocated cores. Of course, for each task type mapped to hardware at least one core of this type needs to be allocated. However, if too many cores are placed onto an ASIC or FPGA, the available area is exceeded and an area penalty is introduced (line 6). On the other hand, if multiple tasks of the same type are mapped to the same hardware component and the hardware area is not violated, it is possible to implement cores multiple times. In our approach, we allocated additional cores (line 5) for parallel tasks with low mobility (line 4), therefore, the chance to exploit application parallelism is increased. Clearly, from an energy point of view, this is also preferable, especially in the presence of DVS, where a decreased execution time can be exploited. At this point it is possible to compute the static power consumption of the implementation (line 7), taking into account component shut-down. Components can be shut-down during the execution of a certain mode, whenever no tasks belonging to that mode are mapped onto these components. Another aspect is the reconfigurability of FPGAs which allows to exchange the cores to suit the active mode. However, this reconfiguration during a mode change takes time, hence, we introduce a transition penalty if the maximal transition times are exceeded (line 8). Having determined the cores to be implemented (line 5), it is now possible to schedule each mode of the application and to derive a feasible communication mapping (line 10). Since the modes are mutually exclusive, it is possible to employ a communication mapping and scheduling optimisation for a single mode system. We utilise the technique outlined in [12]. If timing constraints are violated by the found schedule, a timing penalty is introduced (line 11). Further, based on the communication mapping and scheduling, the dynamic power consumption of the application can be computed, taking into account DVS (line 12). Similarly to the shut-down of PEs, it is also possible to switch-off a CL when no communications are mapped to that link (line 13). Based upon all estimated power consumptions and penalties, a fitness is calculated (line 14) as, $F_{M}=\bar{p} \cdot t p \cdot\left(1+w_{A} \cdot \sum_{\pi \in \mathcal{P}_{v}}\left(a_{\pi}^{U}-a_{\pi}^{\max }\right) /\left(a_{\pi}^{\max } \cdot 0.01\right)\right)$. $\left(w_{R} \cdot \prod_{T \in \Theta_{v}} t_{T} / t_{T}^{\max }\right)$, where the energy consumption $\bar{p}$ is given by Equation (1) and $t p$ introduces a timing penalty. Further, an area penalty is applied for all PEs with area violation $\mathcal{P}_{v}$ by relating used area $a_{\pi}^{U}$ and area constraint $a_{\pi}^{\max }$. Similarly, a transition time penalty is applied for all transitions that exceed their maximal transition time limit $\Theta_{v}$, i.e., transition time $t_{T}$ exceeds maximal allowed transition time $t_{T}^{\max }$. Both area and transition penalty are weighted $\left(w_{A}\right.$ and $\left.w_{R}\right)$ which allows to adjust the aggressiveness of the penalty. Having assigned a fitness to all individuals of the population, they are ranked using linear scaling (line 15). A tournament selection scheme picks individuals (line16) for mating (line 17). The produced offsprings are inserted into the population (line 18). In order to push the GA away from infeasible and low quality design space regions, we apply four new genetic mutation operators (lines 19-22), introduced next.

Shut-down Improvement: To increase the chances of component shut-down, which leads to a reduction of static power consumption, we employ a simple yet effective strategy during the optimisation. Out of the current population randomly picked individuals (probability $2 \%$ was found to lead to good results) are modified as follows. A single mode $O_{x}$ and a non-essential PE $\pi_{a}$ are selected. Non-essential PEs are considered to be PEs that implement task types that have alternative implementations on other PEs, hence, they are not fundamental for a feasible solution. Our goal is to switch-off PE $\pi_{a}$ during the execution of mode $O_{x}$. Therefore, all tasks of mode $O_{x}$ which are mapped to $\pi_{a}$ are randomly re-mapped to the remaining PEs $\left(\mathcal{P} \backslash \pi_{a}\right)$, hence, $\mathrm{PE} \pi_{a}$ can be shut-down during mode $O_{x}$.

Area Improvement: To avoid convergency towards area infeasible solutions we employ a second strategy. If only infeasible area mappings have been produced for a certain number of generations we try to push the search away from this region by randomly mapping hardware tasks onto software programmable PEs. 


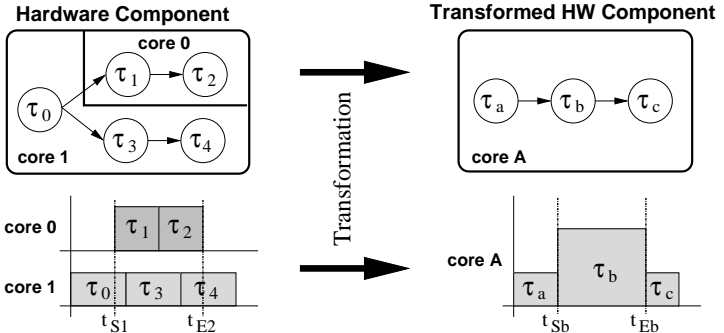

Figure 5. DVS Transformation for HW Cores

Timing Improvement: In contrast to the area improvement strategy, if a certain amount of timing infeasible solutions have been produced, software tasks are randomly mapped to faster hardware implementations. Thereby, the chance to find timing feasible implementations is increased.

Transition Improvement: As mentioned above, cores implemented on FPGAs can be dynamically reconfigured, involving a time overhead. If this overhead exceeds the imposed transition time limits, the mapping is infeasible. Hence, after generating for a certain number of generations solely solutions that violate the transition times, tasks are randomly re-mapped away from the FPGAs that cause the violations.

Although some of the produced genomes might be infeasible in terms of area and timing behaviour, all these strategies have been found to improve the search process significantly by introducing individuals that evolve into high quality solutions.

\subsection{Dynamic Voltage Scaling in Multi-Mode Systems}

Dynamic voltage scaling is a powerful technique to reduce energy consumption by exploiting temporal performance requirements through dynamically adapting processing speed and supply voltage of PEs. The applicability of DVS to embedded distributed systems was demonstrated in $[5,8,10]$. However, these papers concentrated on dynamically changing the performance of software PEs only, while parallel execution of tasks on hardware resources has been neglected. Nevertheless, in the context of energy-efficient multi-mode systems, where performance requirements of each operational mode can vary significantly, DVS needs to be considered carefully. For instance, consider an inverse DCT algorithm, used by the MP3 decoder and the JPEG image decoder, implemented in fast hardware. Clearly, the JPEG decoder should restore images as quickly as possible (maximal clock frequency), while the MP3 decoder works at a fixed sampling rate of $25 \mathrm{~ms}$ (reduced clock). Using DVS it is possible to adapt the HW core speed to suit both needs and reduce the energy dissipation to a minimum.

In this work, we consider that hardware components might employ DVS. However, due to the area and power overhead involved in additional DVS hardware (DC/DC converter), we take into account that all cores allocated to the same hardware component are fed by a single voltage supply, i.e., dynamically scaling the supply voltage simultaneously affects the performance of all cores on that component.

To cope with this, we transform the potentially parallel executing tasks on a single scalable hardware resource into an equivalent set of sequentially executing tasks, taking into account the dynamic power dissipation on each core. Note that this is done to calculate the scaled supply voltages only, i.e., this virtual transformation does not affect the real implementation. Fig. 5 shows the transformation of 5 hardware tasks, executing on two cores, to 3 sequential tasks on a single core. This sequential execution is equivalent to the behaviour of software tasks, hence, a voltage

\begin{tabular}{|c|c|c|c|c|c|}
\hline \multirow{2}{*}{$\begin{array}{c}\text { Example } \\
\text { (No. of } \\
\text { modes) }\end{array}$} & \multicolumn{2}{|c|}{ without probab. } & \multicolumn{3}{|c|}{ with probab. (proposed) } \\
\hline & $\begin{array}{c}\text { Power } \bar{p} \\
(m W)\end{array}$ & $\begin{array}{l}\text { CPU time } \\
(s)\end{array}$ & $\begin{array}{c}\text { Power } \bar{p} \\
(m W)\end{array}$ & $\begin{array}{c}\text { CPU time } \\
(s)\end{array}$ & $\begin{array}{c}\text { Reduc. } \\
(\%)\end{array}$ \\
\hline mul1 (4) & 8.131 & 20.7 & 7.529 & 24.7 & 7.29 \\
\hline mul2 (4) & 3.404 & 15.5 & 2.771 & 18.2 & 18.61 \\
\hline mul3 (5) & 10.923 & 23.4 & 10.430 & 23.0 & 4.17 \\
\hline mul4 (5) & 7.975 & 21.0 & 6.726 & 25.2 & 15.50 \\
\hline mul5 (3) & 5.186 & 18.4 & 4.668 & 22.1 & 10.01 \\
\hline mul6 (4) & 1.677 & 20.6 & 1.301 & 19.9 & 22.46 \\
\hline mul7 (4) & 3.306 & 11.6 & 1.250 & 21.4 & 62.18 \\
\hline mul8 (4) & 1.565 & 32.1 & 1.329 & 28.0 & 15.06 \\
\hline mul9 (4) & 3.081 & 6.0 & 1.901 & 5.8 & 38.28 \\
\hline mul10 (5) & 1.105 & 28.3 & 0.941 & 32.1 & 14.83 \\
\hline mul11 (3) & 2.199 & 9.3 & 1.304 & 16.6 & 40.70 \\
\hline mul12 (4) & 7.006 & 25.4 & 5.975 & 34.2 & 14.69 \\
\hline
\end{tabular}

Table 1. Considering Execution Probabilities (w/o DVS) scaling technique for software processors can be applied.

\section{Experimental Results}

Our co-synthesis approach for energy-efficient multi-mode systems has been implemented on a Pentium III/1.2GHz Linux PC. In order to evaluate its capability to produce high quality solutions in terms of energy consumption, timing behaviour, and hardware area requirements, a set of experiments has been carried out on 12 automatically generated examples (mul1-mul12) and one real-life benchmark (smart phone). All reported results were obtained by running the optimisation processes 40 times and averaging the outcomes.

Each of the 12 generated examples is specified by 3 to 5 operational modes, each consisting of 8 to 32 tasks. The used target architectures contain 2 to 4 heterogeneous PEs, some of which are DVS enabled. These PEs are connected by 1 to 3 CLs.

To illustrate the importance of taking mode execution probabilities into account during the synthesis process, we compare an execution probability neglecting approach with our synthesis technique. Tab. 1 shows this comparison for the 12 generated benchmarks. Columns 2 and 3 give the dissipated average power and optimisation time for the execution probability neglecting approach while Columns 4 and 5 show the same for the proposed approach. Take, for instance, example mul6. Ignoring the execution probabilities during the optimisation an average power of $1.677 \mathrm{~mW}$ is achieved. However, the optimisation under the consideration of the real-world characteristic that modes execute with uneven probabilities the average power can be reduced by an appropriate task mapping and core allocation to $1.301 \mathrm{~mW}$. This is a significant reduction of $22.46 \%$. Furthermore, it can be observed that the proposed technique was able to reduce the energy dissipation of all examples with up to $62.18 \%$. Note that these reductions are achieved without a modification of the underlying hardware architectures, i.e., the system costs are not increased. It is also important to note that the achieved energy reductions are solely introduced by taking the mode execution probabilities into account during the synthesis process, i.e., both compared approaches allow the same resource sharing and rely on the same scheduling technique. Comparing the optimisation times for both approaches it can be observed that the proposed technique shows a slight increased CPU time for most examples, which is mainly due to the design space structure.

We were also interested to see how the proposed technique compares to DVS and if further savings can be achieved by taking the mode probabilities and DVS simultaneously into account. Tab. 2 reports our findings. The DVS technique we use here is an extended version of the one presented in [10]. This extension 


\begin{tabular}{|c|c|c|c|c|c|}
\hline \multirow{2}{*}{$\begin{array}{l}\text { Example } \\
\text { (No. of } \\
\text { modes) }\end{array}$} & \multicolumn{2}{|c|}{ without probab. } & \multicolumn{3}{|c|}{ with probab. (proposed) } \\
\hline & $\begin{array}{l}\text { Power } \bar{p} \\
(\mathrm{~mW})\end{array}$ & $\begin{array}{c}\text { CPU time } \\
(s)\end{array}$ & $\begin{array}{l}\text { Power } \bar{p} \\
(\mathrm{~mW})\end{array}$ & $\begin{array}{c}\text { CPU time } \\
(s)\end{array}$ & $\begin{array}{c}\text { Reduc. } \\
(\%)\end{array}$ \\
\hline mul1 (4) & 4.271 & 526.6 & 3.964 & 768.6 & 10.92 \\
\hline mul2 (4) & 1.568 & 860.4 & 1.273 & 687.4 & 18.82 \\
\hline mul3 (5) & 4.012 & 1053.5 & 3.344 & 1192.2 & 16.66 \\
\hline mul4 (5) & 2.914 & 1135.2 & 2.320 & 1125.4 & 20.39 \\
\hline mul5 (3) & 1.394 & 967.7 & 1.315 & 932.1 & 5.68 \\
\hline mul6 (4) & 0.689 & 472.9 & 0.465 & 593.7 & 32.53 \\
\hline mul7 (4) & 1.331 & 540.3 & 0.479 & 820.7 & 64.02 \\
\hline mul8 (4) & 0.564 & 1262.1 & 0.436 & 1412.0 & 22.64 \\
\hline mul9 (4) & 0.942 & 161.2 & 0.648 & 177.1 & 34.66 \\
\hline mul10 (5) & 0.480 & 1456.3 & 0.394 & 1361.9 & 17.88 \\
\hline mul11 (3) & 0.396 & 318.1 & 0.255 & 403.2 & 35.53 \\
\hline mul12 (4) & 2.857 & 1384.7 & 2.460 & 1450.7 & 13.91 \\
\hline
\end{tabular}

Table 2. Experimental Results with DVS

enables the consideration of DVS not only for software processor but also for parallel executing cores on hardware PEs (see Section 4.2.) As in the first experiments, we compare two approaches, one neglecting the mode execution probabilities during optimisation, while the second takes them into account throughout the synthesis. Similar to Tab.1, Column 2 and 3 of Tab. 2 show the results without consideration of execution probabilities, whilst Column 4 and 5 present the results achieved by the proposed approach. Lets consider again mul6. Although the execution probabilities are neglected in Column 2, we can observe a reduced average power dissipation $(0.689 \mathrm{~mW})$ when compared to the results given in Tab. 1. This clearly demonstrates the high energy reduction capabilities of DVS. Nevertheless, it is possible to further minimise the average power to $0.465 \mathrm{~mW}$ by considering the execution probabilities together with DVS. This is an improvement of $32.53 \%$. For all other benchmarks, savings of up to $64.02 \%$ were achieved. Due to the computation of scaled supply voltages and the influence of scheduling on the energy dissipation, the optimisation times are higher when DVS is considered.

To further validate the proposed co-synthesis technique in terms of real-world applicability, we applied our approach to a smart phone real-life example. This benchmark is based on three publicly available applications: a GSM codec [1], a JPEG decoder [2], and an MP3 decoder [6], Based on these applications, the smart phone offers three different services to the user, namely, a GSM cellular phone, an MP3-player, and a digital camera. Of course, the used applications do not specify the whole smart phone device, however, a major digital part of it. The OMSM for this example is shown in Fig. 1a). For each of the eight operational modes we have extracted the corresponding task graphs from the above given references. These have been software profiled to extract the necessary execution characteristics of each task. On the other hand, the hardware estimations are not based on direct measurements but have been based on realistic assumptions, such that hardware tasks typically executed 5 to 100 times faster than their software counterparts. Depending on the operational mode, the number of tasks and communications varies between 5-88 nodes and 0-137 edges, respectively. The given system architecture consists of one DVS enabled GPP and two ASICs which are connected through a single bus.

Table 3 shows the results of our experiments. Similar to the previous experiments, we compare an execution probabilities neglecting approach with the proposed technique. The first row in Table 3 shows this comparison for a fixed voltage system. Synthesising the system without consideration of execution probabilities, results in an average power of $2.602 \mathrm{~mW}$. Nevertheless,

\begin{tabular}{c||c|c||c|c|c}
\hline \multicolumn{1}{c||}{} & \multicolumn{2}{c||}{ without probab. } & \multicolumn{3}{c}{ with probab. (proposed) } \\
\cline { 2 - 6 } $\begin{array}{c}\text { Smart } \\
\text { phone }\end{array}$ & $\begin{array}{c}\text { Power } \bar{p} \\
(m W)\end{array}$ & $\begin{array}{c}\text { CPU time } \\
(s)\end{array}$ & $\begin{array}{c}\text { Power } \bar{p} \\
(\mathrm{~m} W)\end{array}$ & $\begin{array}{c}\text { CPU time } \\
(s)\end{array}$ & $\begin{array}{c}\text { Reduc. } \\
(\%)\end{array}$ \\
\hline \hline w/o DVS & 2.602 & 80.1 & 1.801 & 96.9 & 30.76 \\
\hline with DVS & 1.217 & 3754.5 & 0.859 & 4344.8 & 29.41 \\
\hline
\end{tabular}

Table 3. Results of Smart Phone Experiments

taking into account the mode usage profile, this can be reduced by $30.76 \%$ to $1.801 \mathrm{~mW}$. We have also applied DVS to this benchmark, considering that the GPP of the given architecture supports DVS functionality (see second row in Table 3 ). We can observe that the power dissipation drops to $1.217 \mathrm{~mW}$, even when neglecting mode execution probabilities. However, the combination of applying DVS and taking execution probabilities into account, results in the lowest power dissipation of $0.859 \mathrm{~mW}$, a $29.41 \%$ reduction. Overall, decreasing the average power from $2.602 \mathrm{~mW}$ to $0.859 \mathrm{~mW}$. This is a significant reduction of nearly $67 \%$.

\section{Conclusions}

We have proposed a novel co-design methodology for energyefficient multi-mode embedded systems. Unlike previous approaches, the presented co-design technique optimises mapping and scheduling not only towards hardware cost and timing behaviour, but additionally reduces power consumption. The key contribution was the development of an effective mapping strategy that considers uneven mode execution probabilities as well as several other important power reduction aspects, such as multiple task implementations. For this purpose, we have introduced a GA based mapping approach along with four improvement strategies that guide the mapping optimisation towards high quality solutions in terms of power consumption, timing feasibility, and area usage. We have validated our approach using several experiments including a smart phone real-life example. These experiments have demonstrated that it is important to take mode execution probabilities into account.

\section{References}

[1] GSM 06.10, Technical University of Berlin. Source code available at http://kbs.cs.tu-berlin.de/ jutta/toast.html

[2] Independent JPEG Group: jpeg-6b. Source code available at ftp://ftp.uu.net/graphics/jpeg/jpegsrc.v6b.tar.gz.

[3] D. E. Goldberg. Genetic Algorithms in Search, Optimization \& Machine Learning. Addison-Wesley, 1989.

[4] T. Grötker, R. Schoenen, and H. Meyr. PCC: A Modeling Technique for Mixed Control/Data Flow Systems. In Proc. ED\&TC 97, March 1997.

[5] F. Gruian and K. Kuchcinski. LEneS: Task Scheduling for Low-Energy Systems Using Variable Supply Voltage Processors. In Proc. ASP-DAC'01, pages 449-455, Jan 2001

[6] J. Hagman. mpeg3play-0.9.6. Source code available at http://home.swipnet.se/ w-10694/tars/mpeg3play-0.9.6-x86.tar.gz.

[7] A. Kalavade and P. A. Subrahmanyam. Hardware/software Partitioning for Multifunction Systems. IEEE Trans. CAD, 17(9):819-837, September 1998.

[8] J. Luo and N. K. Jha. Battery-Aware Static Scheduling for Distributed Real-Time Embedded Systems. In Proc. DAC01, 2001.

[9] H. Oh and S. Ha. Hardware-Software Cosynthesis of Multi-Mode MultiTask Embedded Systems with Real-Time Constraints. In CODES'O2, pages 133-138, May 2002.

[10] M. T. Schmitz and B. M. Al-Hashimi. Considering Power Variations of DVS Processing Elements for Energy Minimisation in Distributed Systems. In Proc. ISSS'01, pages 250-255, October 2001.

[11] M. T. Schmitz, B. M. Al-Hashimi, and P. Eles. Energy-Efficient Mapping and Scheduling for DVS Enabled Distributed Embedded Systems. In Proc. DATE02, pages 514-521, March 2002.

[12] M. T. Schmitz, B. M. Al-Hashimi, and P. Eles. Synthezing Energy-Efficient Embedded Systems with LOPOCOS. Design Automation for Embedded Systems, 6(4):401-424, July 2002.

[13] Y. Shin, D. Kim, and K. Choi. Schedulability-Driven Performance Analysis of Multiple Mode Embedded Real-Time Systems. In Proc. DACOO, pages 495-500, June. 